\title{
ON MATHEMATICAL MODELLING OF TEMPORAL SPATIAL SPREAD OF EPIDEMICS ${ }^{*, * *}$
}

\author{
Kh.A. Khachatryan ${ }^{1}$, A.ZH. NARIMANYAN ${ }^{2, * * *}$ AND \\ A.KH. KHACHATRYAN ${ }^{3}$
}

\begin{abstract}
In the present work a generalized epidemic model containing a system of integraldifferential equations is described. Using different transformations the system is reduced to a single nonlinear multidimensional integral equation. For the obtained equation the existence and uniqueness results are proved. Based on theoretical convergence results several application examples are presented with corresponding numerical results.
\end{abstract}

Mathematics Subject Classification. 92Bxx, 45Gxx.

Received July 11, 2019. Accepted November 25, 2019.

\section{INTROdUCTION}

Presently there are various mathematical models which describe the dynamics of the space-time spread of infectious diseases. Among them a special and important one is the SIR model, which is mainly limited to considering the spread of infectious diseases in time (evolutionary model, [9]). However, due to the high mobility of people, evolutionary models could not provide a real picture of the spread of diseases. A spatially dependent model suggests that an infection at a given point is due to the density of the infection depending on the other points. In turn it means that an arbitrary small infection at any point can become arbitrarily large at any other point $[1-7,10,12-14]$. This effect in the literature is called hair-trigger phenomena [7]. Basic mathematical research for the spatial-and temporal development of the epidemic, within the framework of SI model, was considered by Diekmann in 1978 [7].

The present work is devoted to studying and solving of the problem of the geographical spread of the epidemic within the framework of SIR model.The problem is reduced to a nonlinear multidimensional integral equation. Under certain conditions, the existence and uniqueness results for the equation are proved in the space of monotonic continuous and bounded functions. The paper includes several test examples with corresponding numerical results.

\footnotetext{
* The first and the third authors thank the GKN MON RA for the support in the framework of the project No SCS 18T-1A004.

** The authors are indebted to the referees for their valuable suggestions and a careful reading of the paper.

Keywords and phrases: Epidemics, threshold condition, nonlinear integral equation, existence and uniqueness.

${ }^{1}$ Institute of Mathematics of National Academy of Sciences, Yerevan, Armenia.

2 Department of Mathematics and Computer Science, University of Bremen, Germany.

3 Department of Mathematics, Armenian National Agrarian University, Yerevan, Armenia.

*** Corresponding author: arsen@uni-bremen.de
} 


\section{EPIDEMIC SPATIAL-TEMPORAL SIR MODEL}

The mathematical modelling of epidemics tries to describe the propagation of infectious diseases in some population. Consider a population of $N$ individuals living in $\mathbb{R}^{3}$. Let us assume that there is a infectious disease that spreads through the contacts between individuals. This disease is such that an individual either recovers and becomes immune or dies from it. Taking into consideration the fact that the propagation of the disease can depend on time and space, we divide the population as usual into three groups:

(1) In the first group $S$ are the susceptibles, who are not sick yet and have no immunity to the disease. Let $S(t, x)$ be the density of these individuals at time $t$ and position $x$.

(2) The group I contains all individuals, who are currently infected and can transmit it to the ones from the group $S$. With $I(t, x)$ we denote the density of infected individuals at time $t$ and position $x$.

(3) The rest of the population are in the group $R$ (removed). The members of this group are either recovered and immune, isolated or dead. Let $R(t, x)$ be the density of members of the group $R$.

In addition we assume that the total population size $N$ is large and constant. It is obvious that

$$
S(t, x)+I(t, x)+R(t, x)=1, \text { for all } t \in \mathbb{R}^{+}:=[0,+\infty), x \in \mathbb{R}^{3} .
$$

The basic problem of epidemics is to describe how a group of individuals with an infection spreads the disease to a healthy population depending on time and space. The model we are going to construct is known in the literature as a SIR model and is discussed by several authors [1, 9]. In [7] Diekmann develops a model which involve only two groups of individuals, namely $S$ and $I$ (SI model). Inspired by his technique, we construct now a SIR model for temporal spatial spread of epidemics.

Before we write down the model equations, let us pose some important assumptions. We assume that

(a) every pair of individuals has the same probability of getting into contact (homogeneous mixing),

(b) the infection rate of susceptibles is proportional to the number of contacts between individuals of $S$ and $I$ and depends on $t$ and $x$. We denote the coefficient of proportionality by $B(t, x)$.

(c) individuals are removed from the group $I$ (immune, isolated or dead) with a constant rate $a$.

Let $i(t, \tau, x) \mathrm{d} \tau$ be the density of infectives, which were infected some time between $t-\tau$ and $t-\tau+\mathrm{d} \tau$ at the position $x \in \mathbb{R}^{3}[7]$ : Then

$$
I(t, x)=\int_{0}^{\infty} i(t, \tau, x) \mathrm{d} \tau=\int_{0}^{\infty} i(t-\tau, x) \mathrm{d} \tau=\int_{-\infty}^{t} i(z, x) \mathrm{d} z .
$$

Denote by $S_{0}(x), I_{0}(x)$ and $R_{0}(x)$ the values of the functions $S, I$ and $R$ at initial time $t=0$. Taking into account above mentioned assumptions and definitions, the mathematical model of epidemics can be written as follows:

$$
\begin{gathered}
\frac{\partial S(t, x)}{\partial t}=-B(t, x) S(t, x), \\
\frac{\partial I(t, x)}{\partial t}=B(t, x) S(t, x)-a I(t, x), \\
\frac{\partial R(t, x)}{\partial t}=a(1-S(t, x)-R(t, x)), \quad a>0, \\
S(0, x)=S_{0}(x), \\
i(t-\tau, 0, x)=i(t, \tau, x), \quad i(0, \tau, x)=i_{0}(x) \delta(\tau), \\
R(0, x)=R_{0}(x),
\end{gathered}
$$


where $\delta$ is the Dirac delta function, which means that at time $t=0$ a fresh infection is instantaneously occurred in the population. The infectivity $B(t, x)$ represents the rate at which susceptibles become infective. Adopting the integral form of $B(t, x)$ introduced by Atkinson and Reuter [3] and later generalized by Diekmann [7], we define the infectivity function as

$$
B(t, x)=\int_{\mathbb{R}^{3}} \int_{0}^{\infty} i(t-\tau, y) A(\tau, x, y) \mathrm{d} \tau \mathrm{d} y,
$$

where $A(\tau, x, y)$ is a probability density function that describes the infectivity at position $x \in \mathbb{R}^{3}$ due to one infective individual who has been infected at time $\tau$ and located at $y \in \mathbb{R}^{3}$. The function $A(\tau, x, y)$ couples the duration of illness $\tau$ and the space variables $x$ and $y$. In the present work we follow the Diekmann's assumption stating that there is no coupling, i.e.

$$
A(\tau, x, y)=L(\tau) K(x-y),
$$

where the function $K$ depends on the distance only and represents spatial probability density.

As for the function $L$, it represents the transmission rate depending on the duration of illness $\tau$. Thus, the integral form of the infectivity $B(t, x)$ takes the form

$$
B(t, x)=\int_{\mathbb{R}^{3}} \int_{0}^{\infty} i(t-\tau, y) L(\tau) K(x-y) \mathrm{d} \tau \mathrm{d} y .
$$

Note that the choice of $B(t, x)$ makes the SIR model (2.3)-(2.8) nonlinear.

Since the population is large, we may further assume that the functions $S, I$, and $R$ are continuously differentiable real-valued functions of their arguments.

Next step is to derive an integral equation which is equivalent to the initial value problem (2.3)-(2.8). From (2.1) and (2.2) follows that

$$
i(t, 0, x)=-\frac{\partial S(t, x)}{\partial t}-\frac{\partial R(t, x)}{\partial t} .
$$

Using the equations (2.1), (2.10) and (2.11), the equation (2.3) can be written as

$$
\begin{aligned}
\frac{\partial S(\tilde{t}, x)}{\partial \tilde{t}}= & S(\tilde{t}, x) \int_{0}^{\tilde{t}} \int_{\mathbb{R}^{3}}\left[\frac{\partial S(\tilde{t}-\tau, y)}{\partial \tilde{t}}+\frac{\partial R(\tilde{t}-\tau, y)}{\partial \tilde{t}}\right] L(\tau) K(x-y) \mathrm{d} y \mathrm{~d} \tau \\
& -S(\tilde{t}, x) L(\tilde{t}) \int_{\mathbb{R}^{3}} i_{0}(y) K(x-y) \mathrm{d} y .
\end{aligned}
$$

We integrate the equation (2.12) with respect to $\tilde{t}$ on $(0, t)$. Interchanging the order of integration, we get

$$
\ln \frac{S(t, x)}{S_{0}(x)}=\int_{0}^{t} \int_{\mathbb{R}^{3}}\left[S(\tau, y)-S_{0}(y)+R(\tau, y)-R_{0}(y)-i_{0}(y)\right] L(t-\tau) K(x-y) \mathrm{d} y \mathrm{~d} \tau .
$$

From (2.5) and (2.8) it is easy to verify that

$$
R(t, x)=R_{0}(x) e^{-a t}+a \int_{0}^{t} e^{-a(t-\tau)}(1-S(\tau, x)) \mathrm{d} \tau .
$$


Denoting $u(t, x):=-\ln \frac{S(t, x)}{S_{0}(x)}$, substituting (2.14) in (2.13) and taking into account the equality

$$
R_{0}(x)-R_{0}(x) e^{-a t}=a R_{0}(x) \int_{0}^{t} e^{-a(t-\tau)} \mathrm{d} \tau
$$

we obtain

$$
\begin{aligned}
u(t, x)= & \int_{0}^{t} \int_{\mathbb{R}^{3}}\left[1-R_{0}(y)-S_{0}(y) e^{-u(\tau, y)}\right] L(t-\tau) K(x-y) \mathrm{d} y \mathrm{~d} \tau \\
& +\int_{0}^{t} \int_{\mathbb{R}^{3}} L(t-\tau) K(x-y) \mathrm{d} y\left[a \int_{0}^{\tau}\left[R_{0}(y)-1+S_{0}(y) e^{-u\left(\tau^{\prime}, y\right)}\right] e^{-a\left(\tau-\tau^{\prime}\right)} \mathrm{d} \tau^{\prime}\right] \mathrm{d} \tau .
\end{aligned}
$$

Interchanging again the order of integration in the last integral, we get

$$
u(t, x)=\int_{0}^{t} \int_{\mathbb{R}^{3}}\left[1-R_{0}(y)-S_{0}(y) e^{-u(\tau, y)}\right] \mathcal{L}(t-\tau) K(x-y) \mathrm{d} y \mathrm{~d} \tau,
$$

where

$$
\mathcal{L}(t):=L(t)-a e^{-a t} \int_{0}^{t} e^{a z} L(z) \mathrm{d} z
$$

\section{Existence And uniqueness of the solution of the INTEGRAL EQUATION}

Our main goal in this chapter is to study the issues of solvability of the equation (2.17). Suppose that

$$
\inf _{y \in \mathbb{R}^{3}} S_{0}(y)=s_{0}, \quad \inf _{y \in \mathbb{R}^{3}} R_{0}(y)=r_{0} .
$$

In addition we assume that the kernel function $K$ satisfies the following conditions:

$$
K(x) \geq 0, x \in \mathbb{R}^{3} \text { and } \int_{\mathbb{R}^{3}} K(x) \mathrm{d} x=1 .
$$

Before we prove the main result, we first show that the function $\mathcal{L}$ from (2.18) satisfies a conservativity condition.

Lemma 3.1. Let $L(t)$ be a monotonically increasing bounded and positive function on $\mathbb{R}^{+}$. Let $\eta-L \in L_{1}\left(\mathbb{R}^{+}\right)$, and $\lim _{t \rightarrow+\infty} L(t)=\eta$. Then

$-\mathcal{L}(t) \geq 0, \quad t \in \mathbb{R}^{+}$

- $\mathcal{L} \in L_{1}\left(\mathbb{R}^{+}\right), \quad$ i.e. $\quad \mathcal{L}(t) \geq 0, \quad t \geq 0, \quad \gamma=\int_{0}^{\infty} \mathcal{L}(t) \mathrm{d} t<+\infty$.

Proof. We rewrite the relation (2.18) in the form

$$
\mathcal{L}(t)=L(t)-\eta+\eta-a e^{-a t} \int_{0}^{t} e^{a z} L(z) \mathrm{d} z \leq|\eta-L(t)|+\left|\eta-a e^{-a t} \int_{0}^{t} e^{a z} L(z) \mathrm{d} z\right|
$$


and prove that

$$
\eta-a e^{-a t} \int_{0}^{t} e^{a z} L(z) \mathrm{d} z \in L_{1}\left(\mathbb{R}^{+}\right)
$$

First of all it is easy to verify that

$$
a e^{-a t} \int_{0}^{t} e^{a z} L(z) \mathrm{d} z \leq L(t)\left(1-e^{-a t}\right) \leq L(t) \leq \eta, t \in \mathbb{R}^{+}
$$

since $L(t) \uparrow \eta$ as $t \rightarrow+\infty$.

From (3.4) it follows that $\mathcal{L} \geq 0$ and

$$
\eta-a e^{-a t} \int_{0}^{t} e^{a z} L(z) \mathrm{d} z \geq 0, \quad t \in \mathbb{R}^{+}
$$

Let $r>0$ be an arbitrary number. Using the fact that $\eta-L \in L_{1}\left(\mathbb{R}^{+}\right)$, we derive the following estimation:

$$
\begin{aligned}
0 & \leq \int_{0}^{r}\left(\eta-a e^{-a t} \int_{0}^{t} e^{a z} L(z) \mathrm{d} z\right) \mathrm{d} t=a \int_{0}^{r} \int_{0}^{t} e^{-a(t-z)}(\eta-L(z)) \mathrm{d} z \mathrm{~d} t+\eta \int_{0}^{r} e^{-a t} \mathrm{~d} t \\
& \leq \frac{\eta}{a}+a \int_{0}^{r} \int_{0}^{t} e^{-a(t-z)}(\eta-L(z)) \mathrm{d} z \mathrm{~d} t=\frac{\eta}{a}+a \int_{0}^{r}(\eta-L(z)) \int_{z}^{r} e^{-a(t-z)} \mathrm{d} t \mathrm{~d} z \\
& \leq \frac{\eta}{a}+a \int_{0}^{r}(\eta-L(z)) e^{a z} \int_{z}^{\infty} e^{a t} \mathrm{~d} t \mathrm{~d} z \\
& =\frac{\eta}{a}+\int_{0}^{r}(\eta-L(z)) \mathrm{d} z \leq \frac{\eta}{a}+\int_{0}^{\infty}(\eta-L(z)) \mathrm{d} z<+\infty .
\end{aligned}
$$

Taking the limit of both parts of the inequality as $r \rightarrow+\infty$, we obtain that $\mathcal{L} \in L_{1}\left(\mathbb{R}^{+}\right)$and

$$
\int_{0}^{\infty} \mathcal{L}(x) \mathrm{d} x \leq \frac{\eta}{a}+2 \int_{0}^{\infty}(\eta-L(z)) \mathrm{d} z .
$$

Moreover,

$$
\int_{0}^{\infty} \mathcal{L}(t) \mathrm{d} t=\frac{\eta}{a}
$$

Thus, $\mathcal{L} \in L_{1}\left(\mathbb{R}^{+}\right)$and the lemma is herewith proved.

Here are some examples of the functions $L$ and $\mathcal{L}$ :

- L $(t)=\beta, \quad \mathcal{L}(t)=\beta e^{-a t}, \quad a>0$,

- L $(t)=\beta-\alpha e^{-a t}, \quad 0<\alpha<1, a>0, \quad \mathcal{L}(t)=\beta e^{-a t}+\alpha(a t-1) e^{-a t}$,

$-L(t)=\beta-\frac{\alpha}{1+t^{2}}, \quad \alpha<\beta, \quad \mathcal{L}(t)=\beta e^{-a t}-\frac{\alpha}{1+t^{2}}+a \alpha \int_{0}^{t} \frac{e^{-a(t-z)}}{1+t^{2}} \mathrm{~d} z$ 


$$
\begin{aligned}
-L(t) & = \begin{cases}t+\alpha, & \text { if } 0 \leq t \leq \beta, 0<\alpha<\beta \\
\beta, & \text { if } t \geq \beta\end{cases} \\
\mathcal{L}(t) & = \begin{cases}\frac{1}{a}\left(1-e^{-a t}\right)+\alpha e^{-a t}, & \text { if } 0 \leq t \leq \beta \\
\frac{1}{a} e^{-a t}\left(e^{a \beta}-1+\alpha a\right), & \text { if } t \geq \beta .\end{cases}
\end{aligned}
$$

It can be checked that for all examples the following identity holds:

$$
\gamma=\int_{0}^{\infty} \mathcal{L}(t) \mathrm{d} t=\frac{\beta}{a}
$$

In the literature $\gamma$ is called the contact number.

We are now ready to prove the existence and uniqueness result for the solution of the equation (2.17).

Theorem 3.2. Let the conditions (3.1)-(3.2) be fulfilled and let the function $L$ satisfy the conditions of the Lemma above. Then the equation $(2.17)$ has a positive and bounded unique solution $u(t, x)$ in $C\left([0,+\infty) \times \mathbb{R}^{3}\right)$. Moreover, $u(t, x) \uparrow$ in $t$ on $\mathbb{R}^{+}$.

If additionally $R_{0}\left(\mathrm{x}_{1}, \mathrm{x}_{2}, \mathrm{x}_{3}\right)$ and $S_{0}\left(\mathrm{x}_{1}, \mathrm{x}_{2}, \mathrm{x}_{3}\right)$ are monotonously decreasing on $\mathbb{R}$ with respect to $\mathrm{x}_{j},(j=$ $1,2,3)$, then $u\left(t, \mathrm{x}_{1}, \mathrm{x}_{2}, \mathrm{x}_{3}\right) \uparrow$ with respect to $\mathrm{x}_{j}(j=1,2,3)$ on $\mathbb{R}$, and the following limit relation takes place:

$$
\lim _{t \rightarrow+\infty} \lim _{x_{1} \rightarrow+\infty} \lim _{\mathrm{x}_{2} \rightarrow+\infty} \lim _{3} u\left(t, \mathrm{x}_{1}, \mathrm{x}_{2}, \mathrm{x}_{3}\right)=c,
$$

where $c$ is the positive unique root of the algebraic equation

$$
c=\gamma\left(1-r_{0}-s_{0} e^{-c}\right) .
$$

Proof. We consider the following iteration for the equation (2.17):

$$
\begin{gathered}
u_{n+1}(t, x)=\int_{0}^{t} \int_{\mathbb{R}^{3}}\left[1-R_{0}(y)-S_{0}(y) e^{-u_{n}(\tau, y)}\right] \mathcal{L}(t-\tau) K(x-y) \mathrm{d} y \mathrm{~d} \tau, \\
u_{0}=0, \quad n=0,1,2, \ldots
\end{gathered}
$$

Applying the method of mathematical induction, it is easy to show that $u_{n}(t, x) \uparrow$ in $n$. Indeed, the inequality $u_{1}(t, x) \geq u_{0}(t, x)$ is obvious. It follows immediately from the nonnegativity of kernel function $\mathcal{L}(t) K(x-y)$ and the condition $R_{0}(y)+S_{0}(y)<1$.

Assume that $u_{n}(t, x) \geq u_{n-1}(t, x)$ for some $n \in \mathbb{N}$. Denoting

$$
\chi_{y}(u):=1-R_{0}(y)-S_{0}(y) e^{-u} u \in \mathbb{R}
$$

and taking into account the monotonicity of $\chi$ with respect to $u$, we obtain

$$
u_{n+1}(t, x) \geq \int_{0}^{t} \int_{\mathbb{R}^{3}}\left[1-R_{0}(y)-S_{0}(y) e^{-u_{n-1}(\tau, y)}\right] \mathcal{L}(t-\tau) K(x-y) \mathrm{d} y \mathrm{~d} \tau=u_{n}(t, x) .
$$

Now we check that the functions $u_{n}(t, x)$ build a monotonously nondecreasing sequence in $t$. Let $t_{1}>t_{2}$ and $u_{n}(t, x)$ be increasing in $t$ on $\mathbb{R}^{+}$for some $n \in \mathbb{N} \backslash\{0\}$. Then

$$
\begin{aligned}
u_{n+1}\left(t_{1}, x\right)= & \int_{0}^{t_{1}} \int_{\mathbb{R}^{3}}\left[1-R_{0}(y)-S_{0}(y) e^{-u_{n}\left(t_{1}-\tau, y\right)}\right] \mathcal{L}(\tau) e^{-a \tau} K(x-y) \mathrm{d} y \mathrm{~d} \tau \\
& \geq \int_{0}^{t_{2}} \int_{\mathbb{R}^{3}}\left[1-R_{0}(y)-S_{0}(y) e^{-u_{n}\left(t_{2}-\tau, y\right)}\right] \mathcal{L}(\tau) K(x-y) \mathrm{d} y \mathrm{~d} \tau=u_{n+1}\left(t_{2}, x\right) .
\end{aligned}
$$


Next we turn our attention to the characteristic equation

$$
c=\gamma\left(1-r_{0}-s_{0} e^{-c}\right) .
$$

We show first that the equation (3.11) has a positive unique root. For this purpose we introduce the function

$$
\psi(\lambda)=\lambda-\gamma\left(1-r_{0}-s_{0} e^{-\lambda}\right), \quad \lambda \geq 0
$$

and observe that

$$
\psi(0)=\gamma\left(s_{0}+r_{0}-1\right)<0, \quad \psi(+\infty)=\infty, \quad \psi^{\prime}(\lambda)=1-s_{0} \gamma e^{-\lambda}
$$

Here we consider two possibilities (threshold conditions):

(a) $\gamma s_{0}<1$, in this case the population does not suffer under epidemy,

(b) $\gamma s_{0} \geq 1$, we have an epidemy case here.

If $\gamma s_{0} \leq 1$ then the function $\psi(\lambda) \uparrow$ on $[0,+\infty)$.

If $\gamma s_{0} \geq 1$ then $\lambda_{0}=\ln \gamma s_{0}$ is the unique minimum point for the function $\psi(\lambda)$.

From listed properties of the function $\psi(\lambda)$ it follows immediately that the equation (3.11) possesses unique positive solution $c$.

We remark that one can also prove that

$$
u_{n}(t, x) \leq c, n=0,1,2, \ldots,(t, x) \in(0, T) \times \mathbb{R}^{3} .
$$

Indeed, when $n=0$ the inequality (3.14) is obvious. Suppose that (3.14) holds for some $n \in \mathbb{N}$. Using the lemma from above and due to (3.1)-(3.3), (3.14) as well as the monotonicity of the function $\chi(u)$, we obtain

$$
\begin{gathered}
u_{n+1}(t, x) \leq \int_{0}^{t} \int_{\mathbb{R}^{3}}\left(1-R_{0}(y)-S_{0}(y) e^{-c}\right) \mathcal{L}(t-\tau) K(x-y) \mathrm{d} y \mathrm{~d} \tau \\
\leq\left(1-r_{0}-s_{0} e^{-c}\right) \int_{0}^{t} \int_{\mathbb{R}^{3}} \mathcal{L}(t-\tau) K(x-y) \mathrm{d} y \mathrm{~d} \tau \\
\leq \gamma\left(1-r_{0}-s_{0} e^{-c}\right)=c .
\end{gathered}
$$

By induction it is easy to show that

$$
u_{n} \in C\left(\mathbb{R}^{+} \times \mathbb{R}^{3}\right) .
$$

Thus the sequence of functions $\left\{u_{n}(t, x)\right\}_{n=0}^{\infty}$ has a pointwise limit as $n$ goes to infinity,

$$
\lim _{n \rightarrow \infty} u_{n}(t, x)=u(t, x) .
$$

Therefore, in accordance to B.Levi's theorem [11], the limit function $u(t, x)$ satisfies the equation (2.17).

Due to continuity of convolution operation for integrable and bounded function it follows from (2.17) that

$$
u \in C\left(\mathbb{R}^{+} \times \mathbb{R}^{3}\right) .
$$

Hence according to Dini's theorem the sequence $\left\{u_{n}(t, x)\right\}_{n=0}^{\infty}$ converges uniformly to the function $u(t, x)$ on each compact set of $\mathbb{R}^{+} \times \mathbb{R}^{3}$.

Next we discuss the asymptotic behaviour of solution as $t \rightarrow+\infty$ for each fixed $x$, and asymptotic behaviour $u(\infty, x)$ as $x$ tends to infinity. Since $u(t, x) \uparrow$ in $t$ on $[0,+\infty)$, then there exists a function $w(x)$ such that

$$
w(x)=u(+\infty, x)=\lim _{t \rightarrow+\infty} u(t, x) .
$$


Using the transition rule of limits for convolution type operation

$$
\lim _{x \rightarrow \infty} \int_{0}^{x} K(x-t) f(t) \mathrm{d} t=\left(\int_{0}^{\infty} K(t) \mathrm{d} t\right) \cdot \lim _{t \rightarrow \infty} f(t)
$$

and employing again the equation, we obtain

$$
w(x)=\gamma \int_{\mathbb{R}^{3}}\left[1-R_{0}(y)-S_{0}(y) e^{-w(y)}\right] K(x-y) \mathrm{d} y .
$$

We assume that the functions $R_{0}\left(\mathrm{x}_{1}, \mathrm{x}_{2}, \mathrm{x}_{3}\right)$ and $S_{0}\left(\mathrm{x}_{1}, \mathrm{x}_{2}, \mathrm{x}_{3}\right)$ are monotonously decreasing in $\mathrm{x}_{j}$ on $\mathbb{R}$. First note that each function from sequences $u_{n}\left(t, \mathrm{x}_{1}, \mathrm{x}_{2}, \mathrm{x}_{3}\right)$ for each fixed $\left(t, \mathrm{x}_{1}, \mathrm{x}_{2}\right) \in \mathbb{R}^{+} \times \mathbb{R}^{2}$ is monotonously nondecreasing in $\mathrm{x}_{1}$ on $\mathbb{R}$.

In case when $n=0$ this fact immediately follows from definition of zero approximation. Assuming that $u_{n}\left(t, \mathrm{x}_{1}, \mathrm{x}_{2}, \mathrm{x}_{3}\right) \uparrow$ in $\mathrm{x}_{1}$ on $\mathbb{R}$ for some $n \in N$ and using the monotonicity of the function $\chi(u)$ for $\mathrm{x}_{1}>\tilde{\mathrm{x}}_{1}$, $\left(\mathrm{x}_{1}, \tilde{\mathrm{x}}_{1} \in \mathbb{R}\right)$, from $(3.7)$ we get

$$
\begin{aligned}
& u_{n+1}\left(t, \mathrm{x}_{1}, \mathrm{x}_{2}, \mathrm{x}_{3}\right)=\int_{0}^{t} \int_{\mathbb{R}^{3}}\left(1-R_{0}(y)-S_{0}(y) e^{-u_{n}\left(\tau, y_{1}, y_{2}, y_{2}\right)} \mathcal{L}(t-\tau) K(x-y) \mathrm{d} y \mathrm{~d} \tau\right. \\
= & \int_{0}^{t} \int_{\mathbb{R}^{3}}\left(1-R_{0}\left(\mathrm{x}_{1}-z_{1}, \mathrm{x}_{2}-z_{2}, \mathrm{x}_{3}-z_{3}\right)-S_{0}\left(\mathrm{x}_{1}-z_{1}, \mathrm{x}_{2}-z_{2}, \mathrm{x}_{3}-z_{3}\right) e^{-u_{n}\left(\tau, \mathrm{x}_{1}-z_{1}, \mathrm{x}_{2}-z_{2}, \mathrm{x}_{3}-z_{3}\right)}\right) . \\
& \mathcal{L}(t-\tau) K\left(z_{1}, z_{2}, z_{3}\right) \mathrm{d} z_{1}, \mathrm{~d} z_{2}, \mathrm{~d} z_{3} \mathrm{~d} \tau \geq \int_{0}^{t} \int_{\mathbb{R}^{3}}\left(1-R_{0}\left(\tilde{\mathrm{x}}_{1}-z_{1}, \mathrm{x}_{2}-z_{2}, \mathrm{x}_{3}-z_{3}\right)\right. \\
& \left.-S_{0}\left(\tilde{\mathrm{x}}_{1}-z_{1}, \mathrm{x}_{2}-z_{2}, \mathrm{x}_{3}-z_{3}\right) e^{-u_{n}\left(\tau, \tilde{\mathrm{x}}_{1}-z_{1}, \mathrm{x}_{2}-z_{2}, \mathrm{x}_{3}-z_{3}\right)}\right) \mathcal{L}(t-\tau) K\left(z_{1}, z_{2}, z_{3}\right) \mathrm{d} z_{1} \mathrm{~d} z_{2} \mathrm{~d} z_{3} \mathrm{~d} \tau \\
& =u_{n+1}\left(t, \tilde{\mathrm{x}}_{1}, \mathrm{x}_{2}, \mathrm{x}_{3}\right) .
\end{aligned}
$$

Similarly we can prove that $u_{n} \uparrow$ both in $\mathrm{x}_{2}$ and $\mathrm{x}_{3}$. Consequently, the limit function $u\left(t, \mathrm{x}_{1}, \mathrm{x}_{2}, \mathrm{x}_{3}\right)$ is also monotonously nondecreasing in $\mathrm{x}_{j},(j=1,2,3$.) on $\mathbb{R}$.

Obviously the function $w\left(\mathrm{x}_{1}, \mathrm{x}_{2}, \mathrm{x}_{3}\right)$ also has the same property. Since $w(x) \leq c, \quad x \in \mathbb{R}^{3}$, then there exists a number $\theta$ such that

$$
0<\theta=\lim _{\mathrm{x}_{1} \rightarrow+\infty} \lim _{\mathrm{x}_{2} \rightarrow+\infty} \lim _{\mathrm{x}_{3} \rightarrow+\infty} w\left(\mathrm{x}_{1}, \mathrm{x}_{2}, \mathrm{x}_{3}\right) \leq c .
$$

Taking the limits of both parts of (3.22) consistently as $\mathrm{x}_{3} \rightarrow+\infty, \mathrm{x}_{2} \rightarrow+\infty, \mathrm{x}_{1} \rightarrow+\infty$ and using the fact that

$$
\lim _{\mathrm{x} \rightarrow+\infty} \int_{-\infty}^{+\infty} K(\mathrm{x}-t) f(t) \mathrm{d} t=\left(\int_{-\infty}^{+\infty} K(u) \mathrm{d} u\right) \lim _{\mathrm{x} \rightarrow+\infty} f(\mathrm{x})
$$

we get

$$
\theta=\gamma\left(1-r_{0}-s_{0} e^{-\theta}\right) .
$$

Considering the facts that $c \geq \theta>0$ and the characteristic equation (3.11) has a unique positive solution, we conclude that $\theta=c$. 
It remains to prove that the solution of the equation (2.17) is unique, bounded, positive and belongs to the following class of functions:

$$
\mathfrak{M}=\left\{u: u \in C_{M}\left([0, T] \times \mathbb{R}^{3}\right) \text { and } \sup _{x \in \mathbb{R}^{3}} u(x, t) \in C[0, T]\right\} .
$$

Suppose the contrary, let the equation (2.17) have two bounded and positive solutions $u(t, x)$ and $v(t, x)$. We estimate

$$
\begin{gathered}
|u(t, x)-v(t, x)| \leq s_{0} \int_{0}^{t} \int_{\mathbb{R}^{3}}\left|e^{-u(\tau, y)}-e^{-v(\tau, y)}\right| \mathcal{L}(t-\tau) K(x-y) \mathrm{d} y \mathrm{~d} \tau \\
\leq \sup _{\tau \in \mathbb{R}^{+}} \mathcal{L}(\tau) \cdot s_{0} \int_{0}^{t} \sup _{y \in \mathbb{R}^{3}}|u(\tau, y)-v(\tau, y)| \mathrm{d} \tau .
\end{gathered}
$$

Denoting

$$
\alpha(t):=\sup _{x \in \mathbb{R}^{3}}|u(t, x)-v(t, x)|
$$

we get

$$
\alpha(t) \leq \sup _{\tau \in \mathbb{R}^{+}} \mathcal{L}(\tau) \cdot s_{0} \int_{0}^{t} \alpha(\tau) \mathrm{d} \tau .
$$

Gronwall's lemma [8] yields then

$$
\alpha(t) \leq 0, \quad t \in[0, T]
$$

On the other hand,

$$
\alpha(t)=\sup _{x \in \mathbb{R}^{3}}|u(t, x)-v(t, x)| \geq 0 .
$$

Hence $\alpha(t)=0$, and therefore $u(t, x)=v(t, x)$, which means the the equation (2.17) has a unique solution in $\mathfrak{M}$.

\section{NUMERICAL RESUltS}

In this section we present several numerical results, which are not based on real data. We just test the model in several cases with initially given data. All computations are conducted in Matlab.

1. Spatial model, one dimensional case. We start with one dimensional case. As test functions and parameters we take

$$
\begin{gathered}
R_{0}(\mathrm{x})=0, S_{0}(\mathrm{x})=\frac{s_{1}}{1+e^{2 \mathrm{x}}}+s_{0}, K(\mathrm{x})=\frac{1}{\sqrt{\pi}} e^{-\mathrm{x}^{2}}, \mathcal{L}(t)=\beta e^{-a t}, \\
\beta=12, a=2, s_{1}=0.7, s_{0}=0.2 .
\end{gathered}
$$

Observe that

$$
S_{0}(+\infty)=\inf _{\mathrm{x} \in \mathbb{R}} S_{0}(\mathrm{x})=s_{0}
$$



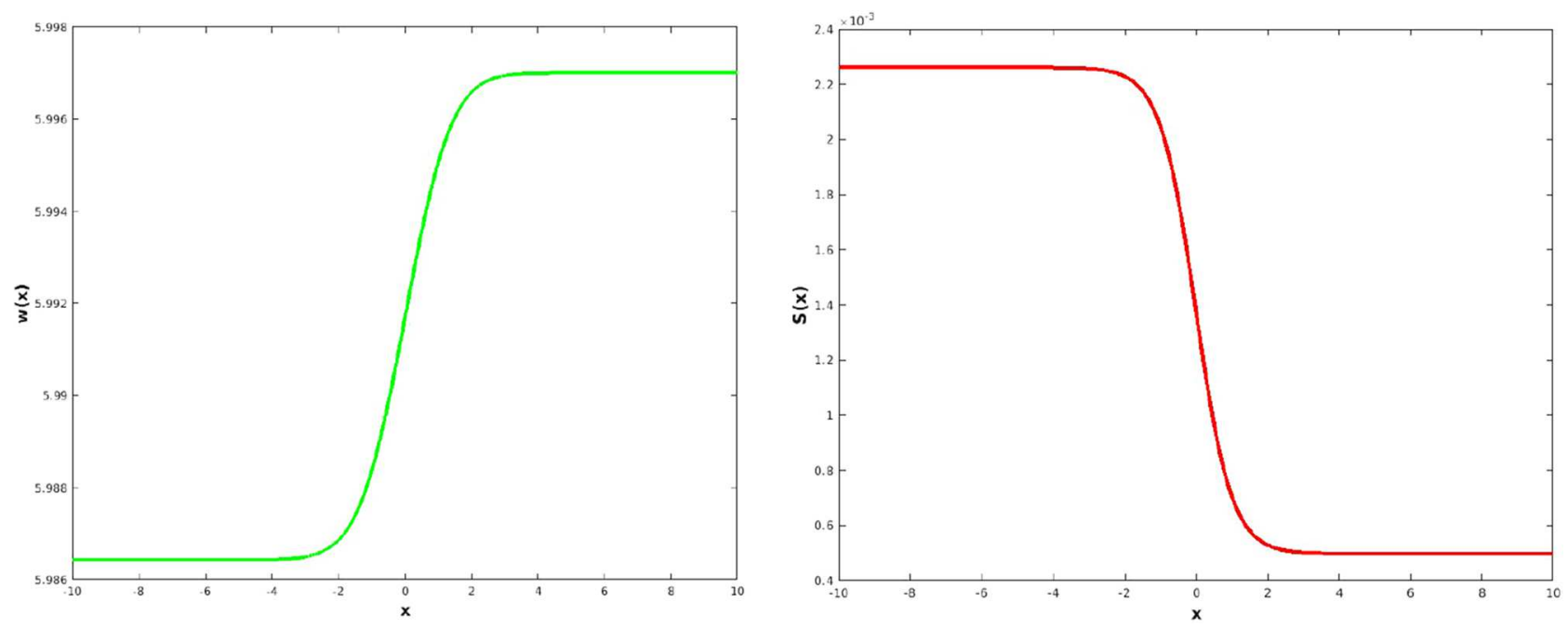

FIgURE 1. The limit function $w(\mathrm{x})$ and propagation function $S(\mathrm{x})$ of susceptibles.

$$
S_{0}(-\infty)=\sup _{\mathrm{x} \in \mathbb{R}} S_{0}(\mathrm{x})=s_{0}+s_{1} .
$$

Let $w(\mathrm{x})=u(+\infty, \mathrm{x}), \mathrm{x} \in \mathbb{R}$, then $S(+\infty, \mathrm{x})=S_{0}(x) e^{-w(\mathrm{x})}$ will be the density of susceptible individuals at location $\mathrm{x}$, who managed to avoid the infection. Obviously, $I(+\infty, \mathrm{x})$ will be the density of infected individuals at position $\mathrm{x}$ after the epidemic is gone.

Finally $R(+\infty, \mathrm{x})=1-S(+\infty, \mathrm{x})-I(+\infty, \mathrm{x})$ represents the number of people at location $x$, who recovered and acquired immunity or died after the epidemic passed. Taking into account the condition (47), the equation (39) for one dimensional case can be written as

$$
w(\mathrm{x})=\frac{\beta}{\sqrt{\pi} a} \int_{\mathbb{R}}\left[1-\left(\frac{s_{1}}{1+e^{2 z}}+s_{0}\right) e^{-w(z)}\right] e^{-(\mathrm{x}-z)^{2}} \mathrm{~d} z .
$$

The equation (4.4) is solved using an iteration technique. Starting with $w_{0}=0, n=0,1,2, \ldots$ we construct a sequence of functions $\left\{w_{n}(\mathrm{x})\right\}_{n=0}^{\infty}$, which converge pointwise. The limit function $w(\mathrm{x})$ is illustrated in Figure 1.

2. Evolutional Model. Now we consider the model in the case when the evolution of the populations depends only on time. We introduce the following notations:

$$
\begin{gathered}
S^{+}(t)=S(t,+\infty)=s_{0} e^{-u^{+}(t)}, \\
S^{-}(t)=S(t,-\infty)=\left(s_{0}+s_{1}\right) e^{-u^{-}(t)}, \\
u^{ \pm}(t)=u(t, \pm \infty) .
\end{gathered}
$$

Here $S^{ \pm}(t)$ are the densities of susceptible individuals at time $t$ at $\mathrm{x}= \pm \infty$ respectively.

Using known limit transition rule for convolution operators from (2.17) we obtain the following simple Voltera type nonlinear integral equation:

$$
u^{+}(t)=\int_{0}^{t}\left[1-s_{0} e^{-u^{+}(t)}\right] \beta e^{-a(t-\tau)} \mathrm{d} \tau
$$



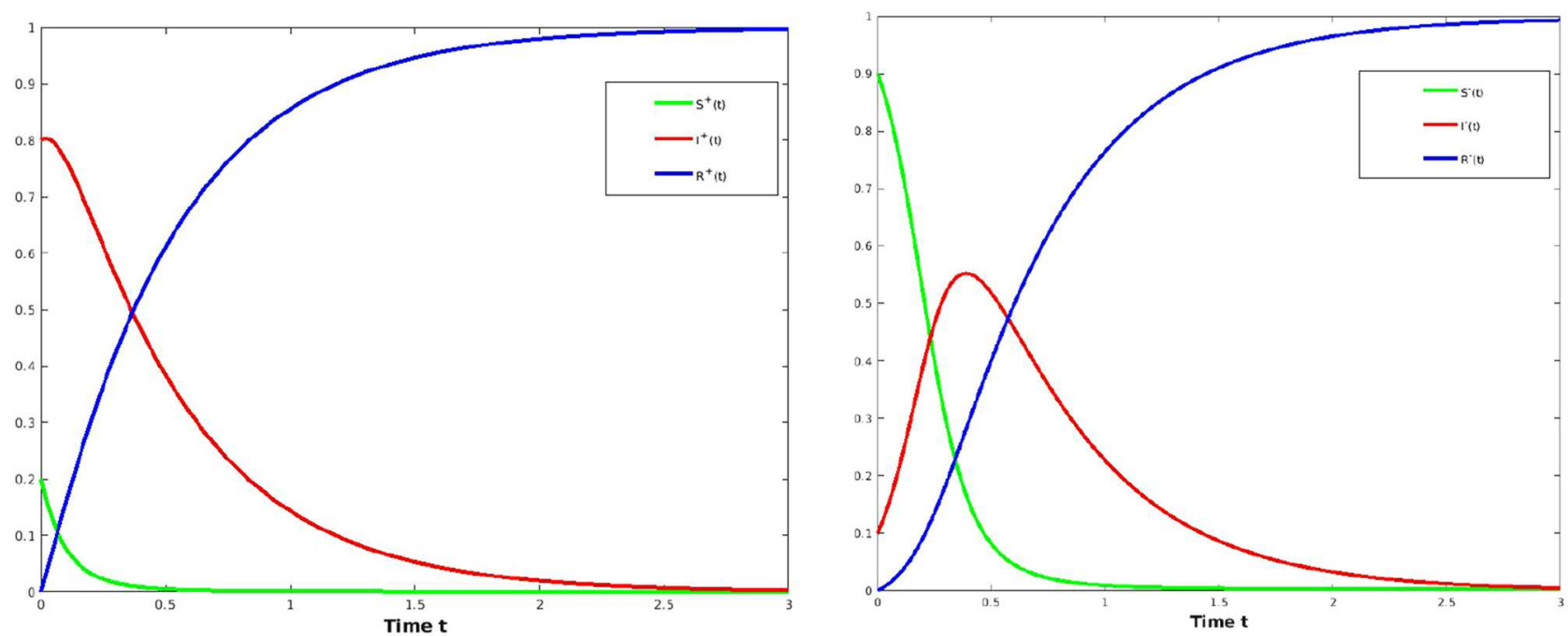

FIGURE 2. The evolution of different groups of individuals.

with characteristic equation

$$
c^{+}-\frac{\beta}{a}\left(1-s_{0} e^{-c^{+}}\right)=0,
$$

where $c^{+}=u(+\infty,+\infty)$. Similarly,

$$
\begin{gathered}
u^{-}(t)=\int_{0}^{t}\left[1-\left(s_{0}+s_{1}\right) e^{-u^{-}(t)}\right] \beta e^{-a(t-\tau)} \mathrm{d} \tau, \\
c^{-}-\frac{\beta}{a}\left(1-\left(s_{0}+s_{1}\right) e^{-c^{-}}\right)=0,
\end{gathered}
$$

where $c^{-}=u(+\infty,-\infty)$. The equations (4.8) and (4.10) can be again solved by iteration technique by using the same parameters as in the first case. The limit functions $S^{ \pm}(t), I^{ \pm}(t)$ and $R^{ \pm}(t)$ are illustrated in Figure 2 .

Note that the functions $I^{ \pm}(t)$ and $R^{ \pm}(t)$ can be computed from the equations below.

$$
\begin{gathered}
I^{+}(t)=I(t,+\infty)=1-\frac{a}{\beta} u^{+}(t)-s_{0} e^{-u^{+}(t)}, \\
I^{-}(t)=I(t,-\infty)=1-\frac{a}{\beta} u^{-}(t)\left(s_{0}+s_{1} e^{-u^{-}}(t)\right), \\
R^{ \pm}(t)=1-S^{ \pm}(t)-I^{ \pm}(t) .
\end{gathered}
$$

One can observe that the number of susceptible individuals decreases as the epidemic proceeds. With decreasing number of susceptible the rate of new infections also decreases. At some time the densities $S^{ \pm}(t)$ are so low, that the rate at which individuals recover exceeds the rate at which new infections occur. Therefore, the densities $I^{ \pm}(t)$ start decreasing as well, i.e. the epidemic ends. 

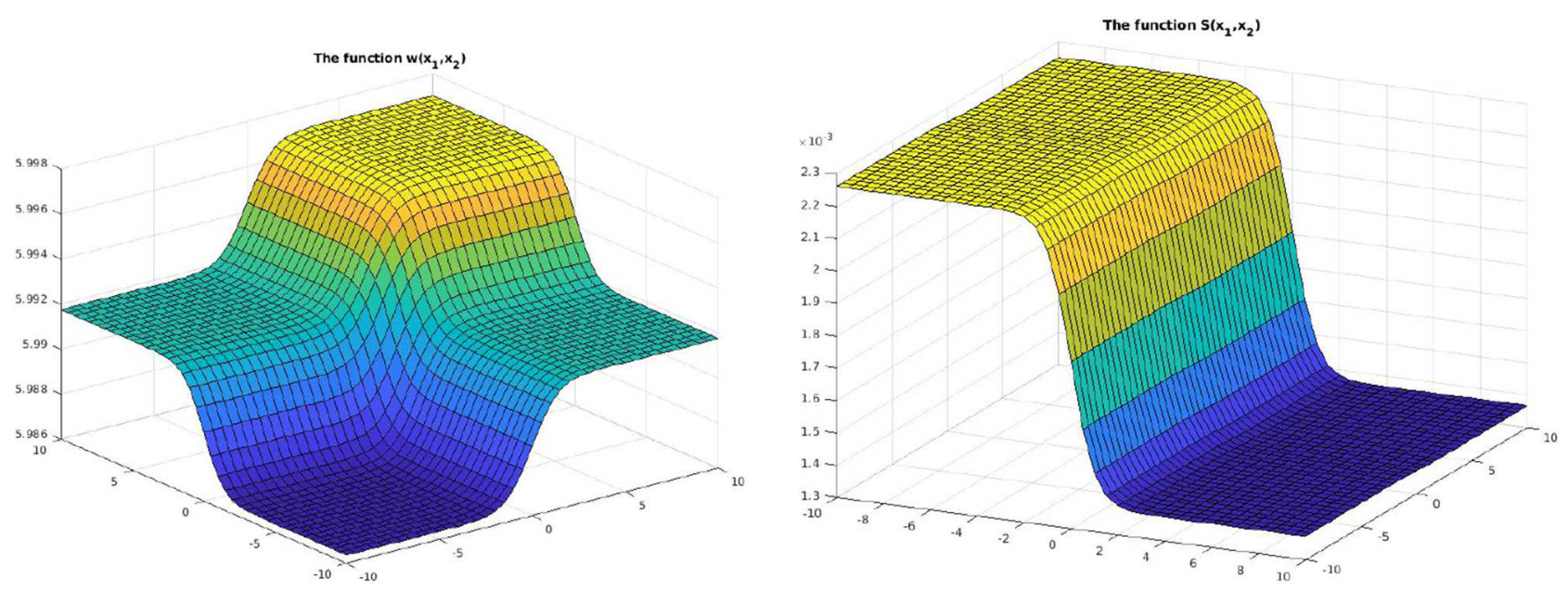

Figure 3. The spatial distribution.

3. Spatial model, two dimensional case. We proceed here similar to one dimensional case and introduce the following test functions:

$$
R_{0}\left(\mathrm{x}_{1}, \mathrm{x}_{2}\right)=0, S_{0}\left(\mathrm{x}_{1}, \mathrm{x}_{2}\right)=\frac{1}{2}\left[\frac{s_{1}}{1+e^{2 \mathrm{x}_{1}}}+2 s_{0}+\frac{s_{1}}{1+e^{2 \mathrm{x}_{2}}}\right], \quad K\left(\mathrm{x}_{1}, \mathrm{x}_{2}\right)=\frac{1}{\pi} e^{-\left(\mathrm{x}_{1}^{2}+\mathrm{x}_{2}^{2}\right)} .
$$

Now consider the following iterative equation:

$$
\begin{gathered}
w_{n+1}\left(\mathrm{x}_{1}, \mathrm{x}_{2}\right)=\frac{\beta}{a \pi} \int_{-\infty}^{\infty} \int_{-\infty}^{\infty}\left[1-S_{0}\left(z_{1}, z_{2}\right) e^{-w_{n}\left(z_{1}, z_{2}\right)}\right] e^{-\left(\mathrm{x}_{1}-z_{1}\right)^{2}-\left(\mathrm{x}_{2}-z_{2}\right)^{2}} \mathrm{~d} z_{1} \mathrm{~d} z_{2}, \\
w_{0}=0, \quad n=0,1,2, \ldots, \quad x \in \mathbb{R}^{2}
\end{gathered}
$$

According to our theoretical investigations, the sequence of iterates $w_{n}\left(\mathrm{x}_{1}, \mathrm{x}_{2}\right)$ must converge to a limit function $w\left(\mathrm{x}_{1}, \mathrm{x}_{2}\right)$. Figure 3 illustrates the limit function $w\left(\mathrm{x}_{1}, \mathrm{x}_{2}\right)$ and the corresponding function $S\left(\mathrm{x}_{1}, \mathrm{x}_{2}\right)$.

4. Temporal Spatial Model. Now we consider a mixed temporal-spatial model. The iterative integral equation to be solved is

$$
u_{n+1}(t, \mathrm{x})=\int_{-\infty}^{\infty} \int_{0}^{t}\left[1-\left(\frac{s_{1}}{1+e^{2 z}}+s_{0}\right) e^{-u_{n}(\tau, z)}\right] \frac{\beta}{\sqrt{\pi}} e^{-a(t-\tau)} e^{-(\mathrm{x}-z)^{2}} \mathrm{~d} z \mathrm{~d} \tau, \mathrm{x} \in \mathbb{R}, t \in \mathbb{R}^{+},
$$

which we have to iterate starting with $u_{0}=0$.

In Figure 4 the graph of limit function $u(t, \mathrm{x})=\lim _{n \rightarrow \infty} u_{n}(t, \mathrm{x})$ as well as the function $S(t, \mathrm{x})$ describing the prorogation of susceptible individuals are presented. Note, that the temporal-spatial distribution of infected individuals can be computed by

$$
I(t, \mathrm{x})=1-S(t, \mathrm{x})-a \int_{0}^{t}(1-S(\tau, \mathrm{x})) e^{-a(t-\tau)} \mathrm{d} \tau .
$$



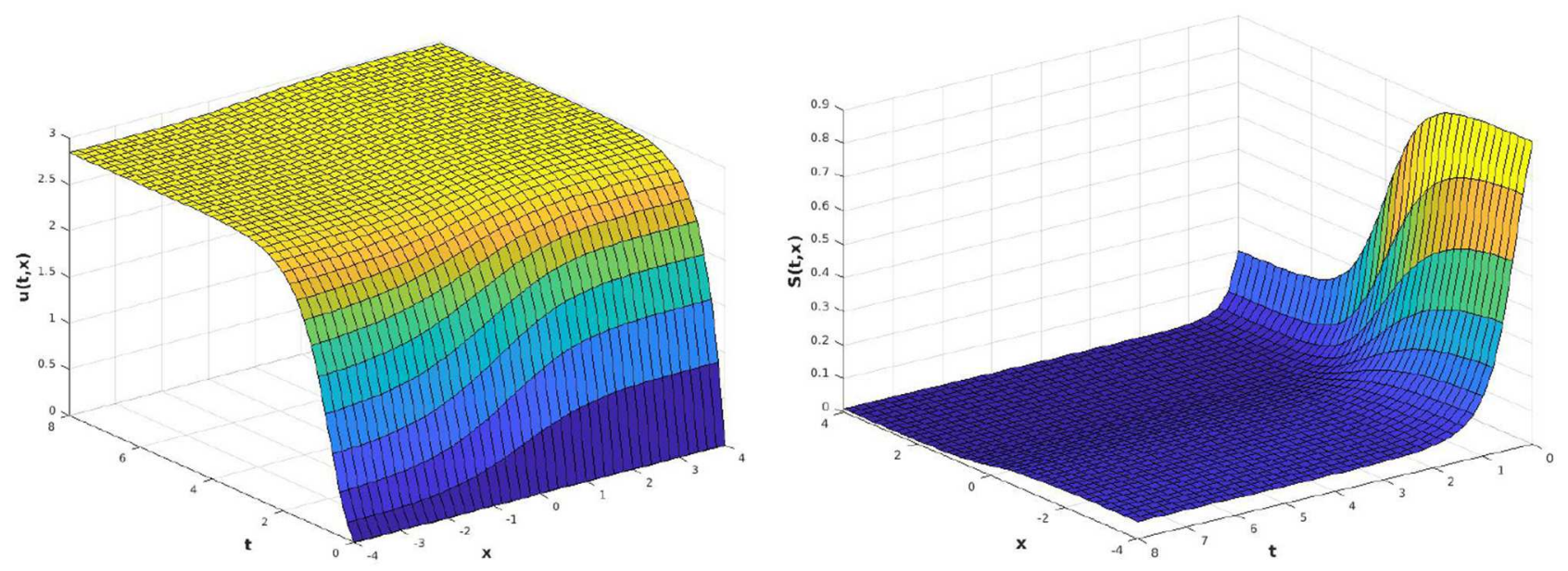

FiguRE 4. The temporal-spatial distribution.

\section{CONCLUDING REMARKS}

The present work discussed the well known model to describe epidemics, the SIR model. We have improved the SI model of Diekmann by involving the density of recovered individuals and added a new equation to Diekmann's model. We now conclude the work by posting some remarks on the contributions of the study and future improvements.

Remark 5.1. The main contribution of the paper is the analysis of a nonlinear integro-differential model by reducing it to a single nonlinear integral equation. The proof of the existence of the solution for such kind of integral equations is not that trivial. We were able to show that the integral equation (2.17) has a unique solution in above defined class of functions $\mathfrak{M}$.

Remark 5.2. Another important contribution of the work is the numerical simulations of the integral equation. The obtained numerical solution allows us to easily describe the propagation of all groups of individuals. The simulation results on temporal, spatial and temporal-spatial dependencies of populations were presented separately.

Remark 5.3. Note that it is quite natural to consider the problem in $\mathbb{R}^{3}$, for example in the case of airborne diseases (influenza, etc.). Nevertheless, the results obtained in this work are applicable for the space $\mathbb{R}^{2}$ and for sphere as well. In the modelling of the spread of infected spores of plants by the wind or the infection of animals in pastures it is natural to consider the space $\mathbb{R}^{2}$ rather than $\mathbb{R}^{3}$.

If the integration is performed over the sphere, the corresponding nonlinear integral operator becomes completely continuous in the space of bounded functions. For such type of operators it is easy to prove the existence of the solution by using the well known classical fixed point principles. If the integration is carried over the space $\mathbb{R}^{3}$ or $\mathbb{R}^{2}$, then the integral operators are not anymore completely continuous. In order to analyze such equations in $\mathbb{R}^{3}$ or $\mathbb{R}^{2}$, it is necessary to develop special approaches. One of the main contributions of this paper is the proof of a constructive theorem for a noncompletely continuous integral operator, as well as the development of a special iteration approach for the numerical solution of the problem.

Finally, if the integration is taken over a sphere whose radius is large enough, then the construction of numerical schemes becomes relatively complicated since the corresponding completely continuous integral operator approaches the zone of noncompletely continuous operators.

Remark 5.4. As for the further steps of the research, the authors plan to

(i) investigate the integral equation (2.17) in the case of general convex nonlinearity, 
(ii) study the stability of the solution of the nonlinear model (2.3)-(2.8) by applying Lyapunov's method (the mehod for hybrid systems is well described in [5] and may be adopted for our model),

(iii) study the temporal-spatial SIR model (2.3)-(2.8) by incorporating the effects of birth, migration and vaccination.

\section{REFERENCES}

[1] R.M. Anderson, Discussion: the Kermack-McKendrick epidemic threshold theorem. Bull. Math. Biol. 53 (1951) 3-32.

[2] R.M. Anderson and R.M. May, Population Biology of Infections. Spring Verlag, Berlin, Heidelberg, New York (1982).

[3] C. Atkinson and G.E. Reuter, Deterministic epidemic waves of critical velocity. Math. Proc. Cambridge Philos. Soc. 80 (1976) 315-330.

[4] V.H. Badshah, P. Porwal, V. Tiwazi, Modelling and Role of dynamics in epidemiology. Int. J. Comput. Sci. Math. 5 (2013) $1-10$.

[5] S. Dashkovskiy and M. Kosmykov, Input-to-state stability of interconnected hybrid systems. Automatica 49 (2013) $1068-1074$.

[6] O. Diekmann, Limiting behavior in an epidemic model. J. Non. Anal. Theory Math. Appl. 1 (1977) 459-470.

[7] O. Diekmann, Thresholds and Travelling Waves for the Geographical Spread of Infection. J. Math. Biol. 6 (1978) 109-130.

[8] T.H. Gronwall, Note on the derivatives with respect to a parameter of the solutions of system of differential equations. Ann. Math 20 (1919) 292-296.

[9] W. Kermack and A. McKendrick, A contribution to the mathematical theory of epidemics. Proc. R. Soc. London A 115 (1927) $700-721$.

[10] A.Kh. Khachatryan and Kh.A. Khachatryan, On solvability of some nonlinear integral equations in problems of spread of epidemics. Proc. Steklov Inst. Math. 306 (2019) 271-287.

[11] A.N. Kolmogorov and S.V. Fomin, Elements of theory functions and functional analyses, Moscow, Nauka, 1981 (in Russian).

[12] L. Rass and J. Radcliffe, Special deterministic epidemics. American Mathematical Society (2003) 273p.

[13] A.G. Sergeev and Kh.A. Khachatryan. On solvability of one class of nonlinear integral equations in spread epidemic problem. Trans. Moscow Math. Soc. 80 (2019) 113-131.

[14] G. Webb, A reaction-diffusion model for a deterministic-diffusive epidemic. J. Math. Anal. Appl. 84 (1981) $150-161$. 